\title{
Die berühmte Kasuistik der Elephantiasis des George Southam anno 1847
}

\section{Zusammenfassung}

Die Elephantiasis galt zu allen Zeiten der Menschheit als beeindruckende Krankheit, die nicht nur Angst und Mitleid, sondern auch ein gewisses Schauern ausgelöst hat. Die Abbildung eines betroffenen Patienten in Büchern und Zeitschriften durfte sich daher eines allgemeinen Interesses gewiss sein. Ein solcher Fall des Londoner Chirurgen George Southam anno 1847 wurde über die Grenzen hinaus bekannt. In Deutschland haben Esmarch und Kulenkampff ihre Monografie Die Elephantiastischen Formen anno 1885 mit hervorragenden Bildtafeln ausgestattet. Das Werk beginnt sogleich mit der Southam'schen Kasuistik. Dabei erscheint es aus medizinhistorischer Sicht interessant, den Gedankengängen zur Pathogenese des Lymphödems von Southam bis Rudolf Virchow zu folgen.

\section{Schlüsselwörter}

Elephantiasis, Lymphödem, Phlegmasia alba dolens, Erysipel, Barbados-Bein

Die extreme Elephantiasis an einer Extremität oder an einem Körperteil erregte zu allen Zeiten der Menschheit Aufmerksamkeit und Ärzte haben nach Erklärungen und Möglichkeiten der Behandlung gesucht. Johann Nepomuk Rust (1775-1840), der erste Chirurg an der Berliner Universitätsklinik, unterschied anno 1832 - wie auch andere Autoren der Zeit - die griechische von der arabischen Form [1, 8, 11]. Darüber hinaus wurden von Rust einzelne Fälle in nördlichen Gegenden wie in Halle gesehen [8].

\section{Die Elephantiasis \\ im 19. Jahrhundert}

Die Elephantiasis Graecorum entsprach der Lepra nodosa. Das Gesicht des Kranken wurde durch Knoten in so arger Weise entstellt, daß die Krankheit den Namen Leontiasis beigelegt hat. An den unteren Extremitäten war vom Elephantenfuß die Rede [8].

Die Elephantiasis Arabum kam in den Ländern Vorderasiens, in Indien und auf den Westindischen Inseln endemisch vor. Man sprach vom „Barbados-Bein“ oder „Cochin-
Bein“ [7, 8]. Über die Ursache der Krankheit war nichts Genaues bekannt, doch sah man Zusammenhänge damit, dass die Eingeborenen ganze Tage und Nächte im sumpfigen Morast auf der Jagd nach Vögeln verbrachten $[1,7]$. Die Geschwulst befällt auch die Gelenke. Die Muskeln verlieren ihre Kraft, und so schleppt der Kranke zuletzt das zu einem unförmigen Klumpen ausgeartete, gelähmte Glied wie eine fremde Last mit sich herum. In therapeutischer Hinsicht haben sich Blutentziehungen durch Scarification, Vesicatoren und Cauterien als schädlich erwiesen. Die besten Erfahrungen brachten Einwickelungen des Beins. Die Amputation war mit aller Vorsicht anzuwenden [8].

Neben diesen tropischen Formen der Elephantiasis mit endemischem Charakter wurden anfangs des 19. Jahrhunderts auch im nördlichen Europa entsprechende Kasuistiken beschrieben, jedoch mit solitärem Auftreten. In Deutschland hat sich besonders Virchow damit befasst [11]. Eine der berühmtesten Krankengeschichten stammt von George Southam aus England. Das Bildnis seiner Patientin, ein Holzstich, wurde über alle Grenzen hinweg reproduziert und demnach weltbekannt [9] (॰ Abb. 1).

\section{Southams Kasuistik anno 1847}

Die unverheiratete 38-jährige Patientin mit phlegmatischem Temperament kam erstmals im Herbst 1843 zur Behandlung. Damals bestand die Krankheit aber schon 20 Jahre lang. Sie begann in ihrem 18. Lebensjahr mit mehreren Schmerzattacken im Bereich des Fußrückens und mit Fieberschüben. Das Ödem blieb bestehen und dehnte sich langsam über das ganze Bein bis in die Leiste aus. Trotzdem war die Patientin in den ersten 8 Jahren nur wenig beeinträchtigt. Dann wiederholten sich die Erysipele häufiger. Aus den Anschwellungen sickerte klare wässrige Flüssigkeit hervor und verwandelte sich zu Inkrustationen mit eitrigen Ansammlungen. Anfangs traten die Schmerzen nur in Intervallen auf und konnten durch Blutegel und Kompressen beherrscht werden, aber nach ein paar Jahren blieben sie konstant und erforderten Opiate.

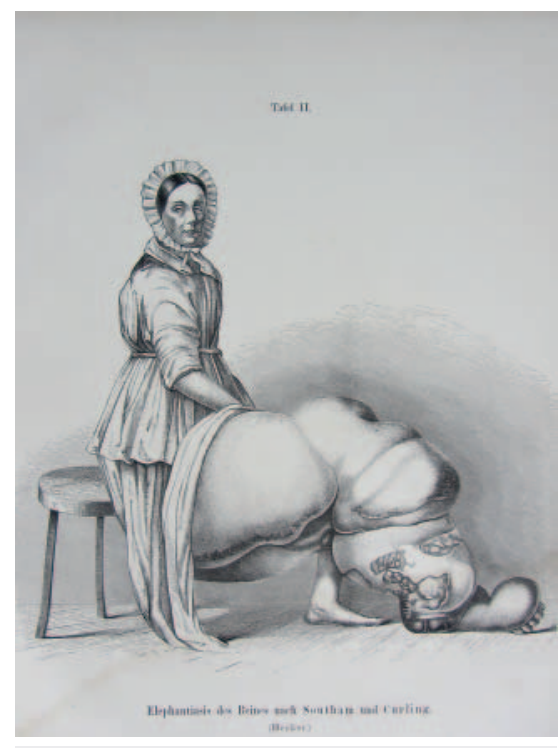

- Abb. 1 Elephantiasis des Beines nach Southam und Curling. Erklärung s. Text. Aus der Monografie von Esmarch und Kulenkamff, Tafel II [1]. Archiv Hach.

In der Familie waren keine besonderen Krankheiten bekannt. Die Patientin selbst berichtete lediglich von der frühen Menopause und einer Unterkühlung als mögliche Ursache der Elephantiasis. Die Haut war am erkrankten Bein feucht und zeigte auf Fingerdruck nicht die geringste Eindellung. Mit einer Lupe konnte man Flüssigkeit austreten sehen. An den Rändern bildeten sich dicke Krusten. Nur die Fußsohle blieb frei.

Bis vor wenigen Wochen konnte die Patientin noch ihre Hausarbeit verrichten. Sie verstarb am 21. November 1847 infolge Dysenterie. Wenige Tage zuvor hatten die Urinsekretion auf 8 Pints/24 Stunden (1 Pint = $1 / 2 \mathrm{I}$ ) und auch die Lymphsekretion am Bein erheblich zugenommen [9].

\section{Sektionsbefunde}

Bei der Obduktion fand sich im subkutanen Bereich des kranken Beins eine speckige Substanz mit eingelagertem Fettgewebe. Knochen, Gelenke und Muskeln erschienen normal. Jedoch waren die Venen deutlich erweitert und die Arterien eher schmal. Zu den Venen schrieb Southam: From the local nature of the pathological changes, the deposit found in the veins would appear not to have been formed 
immediatly from the blood, but from the effusion and consolidation of lymph in their coats, the coagulation of the blood in the folds of the flocculi contributing to fill up the caliber of the vessels [9].

\section{Southams Gedanken zur Pathogenese der Elephantiasis}

Die Ursache der Elephantiasis lag - nach Southams Obduktion - also an einer Erkrankung der Venen, deren Wandung durch Lymphansammlungen aufgetrieben erschien. Sie war „die Konsequenz einer Phlebitis als primäre Affektion oder zumindest als Prädisposition“, hatte jedoch keinen typhoiden oder purulenten Charakter. Die Venen des Uterus und der Beckenregion könnten ebenfalls - im Rahmen einer puerperalen Phlebitis oder einer chronischen Phlegmasia alba dolens - der Anlass gewesen sein. Das gäbe dann auch die Erklärung für den frühen Eintritt der Menopause [9].

Southam fasste seine Theorie der venösen Pathogenese bei entsprechender Veranlagung folgendermaßen zusammen: The albuminous substance, sometimes met in the cellular tissue of infants, presents characters somewhat analogous to what is observed in elephantiasis; the congested state of the veins which usually accompanies it showing, that in all probability it arises from some morbid alteration in them. It would appear, therefore, that the immediate cause and pathological changes of elephantiasis bear an intimate relation to those of phlegmasia dolens, and the induration of the cellular tissue in new-born children; the apparent differences depending on the degrees of venous obstruction and on the remote influences which have originated it [9].

\section{Virchows Theorie anno 1863}

Der Berliner Pathologe Rudolf Virchow (1823-1902) räumte der Elephantiasis anno 1863 im Rahmen seiner Dreißig Vorlesungen über Die krankhaften Geschwülste einen großen Platz ein. Er trennte zunächst - wie andere Autoren - die tropischen Formen der Elephantiasis Graecorum als Lepra und der Elephantiasis Arabum voneinander ab und vermutete, dass hier ein Molluscum contagiosum als Ursache vorliegen könnte.
Eine weitere Form der Elephantiasis, die Rose der „deutschen Schriftsteller aus früherer Zeit“, bezeichnete Virchow als lymphatisches Erysipel. Hierbei spielten die erbliche Disposition bzw. die lymphatische Konstitution eine wichtige Rolle. Als sekundäre Ursachen kamen auch die Phlegmasia alba oder eiternde Bubonen in Betracht. Virchow schrieb dazu: Die venöse Stauung führt von der venösen Hyperämie zum Oedem und damit zu einer gesteigerten Lymphströmung oder, wie man gewöhnlich sagt, zu einer vermehrten Thätigkeit der Lymphgefässe. Die letzteren erweitern, ihre Drüsen vergrössern sich. Die ödematösen Theile disponiren in hohem Masse zu erysipelatösen Entzündungen. Ist der Reiz stark, so nimmt dieses Erysipel den phlegmonösen Charakter an und geht in schlechte Eiterung oder Brand über. Bei geringer Reizung dagegen geschieht etwas ähnliches, wie in den primär und ursprünglich mit Erysipel auftretenden Formen [11].

Wie dem englischen Chirurgen George Southam fiel auch Virchow die besondere Beschaffenheit des interstitiellen Gewebes auf. Es befindet sich fast ganz in dem gallertartigen Zustande des Erysipelas gelatinosum. Das Bindegewebe steckt voll von albuminöser Flüssigkeit. Vielleicht hängt dieser Zustand mit der Störung der Lymphcirculation zusammen. Virchow zitiert dabei eine Reihe von Beobachtungen, dass der ausgetretene Saft eine milchige Farbe hat und wesentliche Bestandtheile der Milch, nehmlich Butter, Käse und Milchzucker enthält [11].

\section{Virchows Kasuistik anno 1861}

Einen ausgezeichneten Fall der Elephantiasis ulcerosa habe ich erst in diesem Winter untersucht, ein amputiertes Bein ( $\bullet$ Abb. 2). Es handelte sich um einen 17-jährigen, in seiner ganzen Entwicklung sehr zurückgebliebenen Menschen, der seit seinem 3. Lebensjahre an mancherlei Knochen- und Gelenkentzündungen gelitten hatte. Ein Geschwür an der vorderen Fläche des Unterschenkels, welches längere Zeit bestanden hatte, war 4 Jahre vor der Amputation geheilt. Ein halbes Jahr später Verstauchung des Fussgelenkes, seitdem zunehmende Anschwellung und Verdickung. Seit 2 Jahren Aufbruch und Ulceration. Die grösste Ausbildung der Anschwellung ist am Fussrücken und an den Zehen zu finden. Letztere sind zu unförmlichen, höckerigen Körpern ausgewachsen. Am grossen Zehen liegt ein

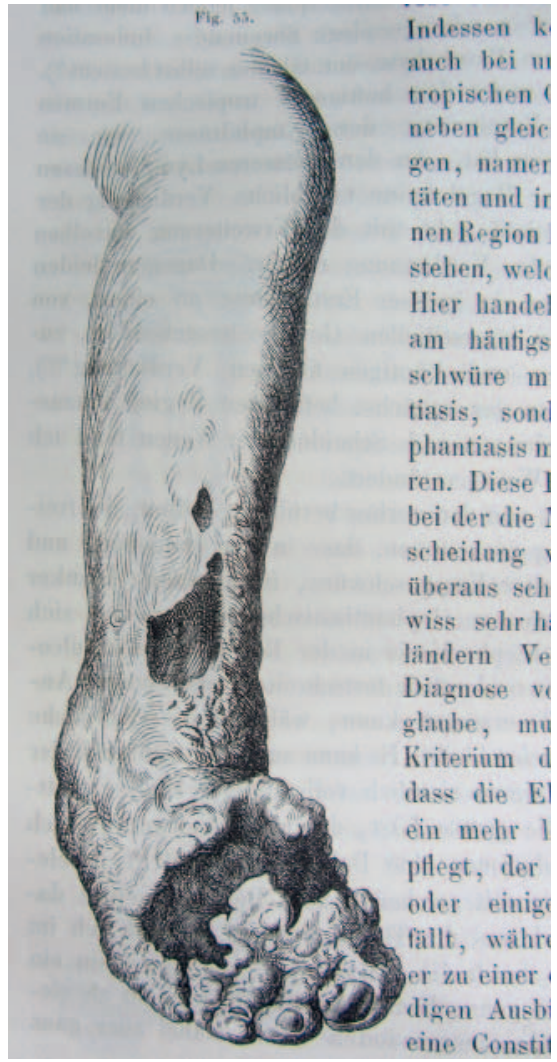

- Abb.2 Elephantiasis dura ulcerosa pedis. Amputationspräparat von Virchow. Erklärung s. Text. Aus Virchows 13. Vorlesung über Die krankhaften Geschwülste [12]. Archiv Hach.

grosses Geschwür mit glattem, harten Grunde und hoch aufsteigenden schwieligen Rändern. Schon von der Mitte des Unterschenkels an verschwindet der Panniculus adiposus in einer weissen, knorpelharten Schwiele, welche von der Haut bis zu den Knochen reicht. Präparat No. 148 vom Jahre 1861.

Virchows Einordnung dieses Falls unter die Rubrik „Elephantiasis“ zeigt, dass seine Ansichten zur Pathogenese bereits in großen Zügen unserer heutigen Vorstellung ähneln [5].

\section{Behandlung der Elephantiasis im 19. Jahrhundert}

Die Verhütung der Krankheit gelang nicht, weil in der Regel mehrere Umstände des täglichen Lebens an der Entstehung beteiligt waren [1] (Kasten 1). Für die Behandlung stand immerhin eine Reihe von Maßnahmen zur Verfügung [1] (Kasten 2). 
Den lokalen Anwendungen kam sowohl von Seiten der Volksmedizin wie auch als ärztliche Handlung die größte Bedeutung zu. Sie reichten von der vertikalen Suspension, den Einwicklungen und dem Gebrauch von faradischem Strom bis zu Blutentziehungen, Skarifikationen und Lymphaderlässen. Die Einreibungen erfolgten mit Merkur- und Jodsalben. Dazu gab es eine Milchdiät (s. o.) und Medikamente wie Digitalis oder Ergotin [1].

\section{KASTEN 1 [1]}

\section{Auslösende Ursachen der Elephan- \\ tiasis}

Wirkung der Schwere

- Stehen, Umhergehen, schwere Arbeit

\section{Kleine Verletzungen}

Phlogogene und infectiöse Stoffe

- Unreinlichkeiten des Patienten

- Absonderungen auf Geschwüren

\section{KASTEN 2 [1]}

\section{Prinzipien der Therapie der Elephant-} hiasis

Ausschaltung allgemeiner Störungen

- Herzfehler, Varizen, Fettsucht, Anämie

Beseitigung chronische Reizzustände der Haut, Unterhaut

- Geschwüre, Ausschläge, Verwachsungen

Förderung des Abflusses der Säfte und des Blutes

Verminderung der Zuflüsse

Verkleinerung der Gewebsmassen

- Ausschneidungen, resorbierende Mittel

Amputationen

Einige Chirurgen führten die tiefe Skarifikation durch. Dabei handelte es sich um bis in die Musculatur eindringende und 4-6 Zoll (1 Zoll=2,54 cm) lange Einschnitte mit nachfolgenden Umschlägen und Bindeneinwickelungen, deren Lisfranc 20-30 machte (Jacques Lisfranc, 1790-1848; französi-

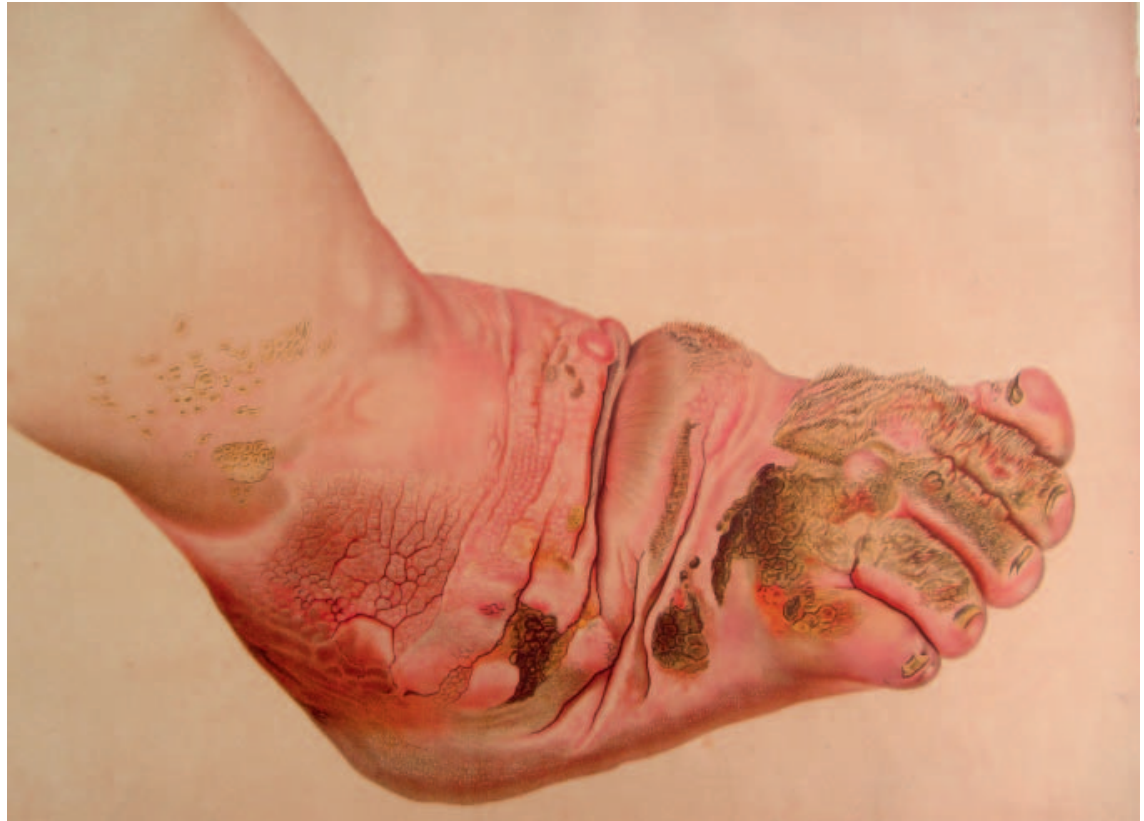

Abb. 3 Elephantiasis pedis. Titeltafel von Esmarch und Kulenkampff [1]. Archiv Hach

scher Chirurg) [1]. Eine Steigerung waren die keilförmigen Exzisionen. Anno 1882 exzidierte Johann von Miculicz (18501905, Breslau) bei einer 18-jährigen Patientin längs des Mittelfussknochens am medialen Fussrande einen $8 \mathrm{~cm}$ langen und $2 \mathrm{~cm}$ breiten Keil und sah danach eine Volumenabnahme des Fusses von $3 \mathrm{~cm}$. Eine entsprechende Operation wurde bereits 1872 von Johann Nepomuk von Nussbaum (1829-1890, München) persönlich an Esmarch mitgeteilt [1].

Eine ganze Epoche der chirurgischen Behandlung der Elephantiasis und sehr widersprüchliche Reaktionen hat die Carnochan'sche Ligatur der A. femoralis anno 1851 ausgelöst $[2,3,6,10]$. Der amerikanische Chirurg Dr. Carnochan führte den Eingriff durch, um durch Reduktion des einfließenden arteriellen Blutvolumens die Zirkulation und die Stoffwechselprozesse im Bein zu reduzieren. In seinem Fall, einem 27-jährigen Einwanderer aus Aachen, führte die Operation zwar zu schweren Komplikationen, war aber zuletzt erfolgreich; zufällig lag in diesem Fall auch eine vaskuläre Dysplasie vor [2, 4].

Die Carnochan'sche Methode fand besonders in Europa eine gewisse Verbreitung, aber die Erfolgsquote blieb enttäuschend [10]. Im Jahr 1869 wurden von Fischer 43 Kasuistiken dokumentiert, davon 2 Todesfälle [3]. Anno 1885 stellten Esmarch und Ku- lenkampff 82 Kasuistiken mit 10 letalen Ausgängen an Pyämie und Gangrän zusammen [1]. Aber es gab auch einzelne Erfolge [4, 6].

\section{Schlussbetrachtung}

Die ausgeprägten Formen der Elephantiasis gingen mit eindrucksvollen klinischen Bildern einher ( $\triangleright$ Abb.3). Die Ärzte vermochten nur wenig mit ihren geringen therapeutischen Möglichkeiten auszurichten. Die Kasuistik des Dr. Southam ist dafür ein klassisches Beispiel. Die Abbildung seiner Patientin ging um die ganze Welt [1, 2, 12]. Die voluminösen Gewebsveränderungen der Elephantiasis betrafen vor allem die untere Extremität, kamen aber auch an den Geschlechtsorganen und anderen Körperteilen vor. In den Tropen lag der Krankheit eine bakterielle oder parasitäre Infektion zugrunde, was auch in den Bezeichnungen Barbados-Bein oder Cochin-Bein zum Ausdruck kam [7, 8]. Auf die kausale Beziehung der Elephantiasis in unseren Breiten zur erblichen Disposition und zum rezidivierenden Erysipel hat Rudolf Virchow hingewiesen [11].

In ihrer eindrucksvollen Monografie Elephantiasis anno 1885 haben Esmarch und Kulenkampff den Patienten und ihrer schlimmen Krankheit ein bemerkenswertes Denkmal gesetzt [1]. Anhand der verschiedenen, sehr eindrücklichen Bildtafeln 
zeigen die Autoren auch, welche Probleme der Differenzialdiagnose zu ihrer Zeit bestanden haben.

\section{Eine verzweifelte Krank- heit will eine verwegene Arznei}

(Altes deutsches Sprichwort)

Interessenkonflikt

Die Autorinnen/Autoren geben an, dass kein Interessenkonflikt besteht.

\section{Autorinnen/Autoren}

Wolfgang Hach

\section{Korrespondenzadresse}

Prof. Dr. Wolfgang Hach

Tacitusstr. 66

60439 Frankfurt am Main

hach.frankfurt@gmx.de

\section{Literatur}

[1] Esmarch F, Kulenkampff D. Die elephantiastischen Formen. Hamburg: Richter; 1885

[2] Carnochan JM. Elephantiasis Arabum of the right inferior extremity, successfully treated by Ligature of the femoral artery. New York J Med 1852; 5: 162-169

[3] Fischer G. Die Behandlung der Elephantiasis Arabum mittelst Ligatur oder Compression der Hauptarterie. Arch Path Anat Physiol Klin Med 1869; 46: 328-350

[4] Hach W. Die Carnochan'sche Operation bei der Elephantiasis im 19. Jahrhundert. Gefässchirurgie im Druck

[5] Hach W, Mumme A, Hach-Wunderl V. VenenChirurgie. Stuttgart: Schattauer; 2013

[6] Leisrink H. Unterbindung der Art. cruralis wegen Elephantiasis cruris. D Zeitschr Chir 1874; 3: 9-16

[7] Lendy J, Rollo J. Über die Drüsenkrankheit in Barbados oder über Wilhelm Hillary's Elephantiasis. Aus dem Englischen übersetzt. Frankfurt am Main: Andreäische Buchhandlung; 1788
[8] Rust JN. Theoretisch-praktisches Handbuch der Chirurgie mit Einschluss der syphilitischen und Augen-Krankheiten. Bd. II, 581; Bd. VI 158 Enslin in Berlin und Gerold in Wien; 1832

[9] Southam G. Case of Elephantiasis. Med Chir Trans 1847; 30: 69-76

[10] Trendelenburg F. Die ersten 25 Jahre der Deutschen Gesellschaft für Chirurgie. Berlin : Springer; 1923: S. 139

[11] Virchow R. Die krankhaften Geschwülste. Bd I, 295 Berlin: Hirschwald; 1863

[12] Vogt H. Das Bild des Kranken. München: Lehmann; 1969: S. 72

Bibliografie

Phlebologie 2021; 50: 8-12

DOI 10.1055/a-1131-4540

ISSN 0939-978X

(c) 2021. Thieme. All rights reserved.

Georg Thieme Verlag KG, Rüdigerstraße 14,

70469 Stuttgart, Germany 